\title{
Strain distribution in GaN/AIN quantum-dot superlattices
}

\author{
E. Sarigiannidou, ${ }^{\text {a) }}$ E. Monroy, B. Daudin, and J. L. Rouvière \\ Département de Recherche Fondamentale sur la Matière Condensée/SP2M, CEA-Grenoble, \\ 17 rue des Martyrs, 38054 Grenoble Cedex 9, France
}

\author{
A. D. Andreev \\ Department of Physics, University of Surrey, Guildford GU2 7XH, United Kingdom
}

(Received 13 June 2005; accepted 8 September 2005; published online 10 November 2005)

\begin{abstract}
The two-dimensional strain distribution in a GaN/AlN quantum-dot (QD) superlattice is measured from high-resolution transmission electron microscopy images using the geometrical phase analysis. The results are compared to elastic theoretical calculations using a combination of Fourier transform and Green's function techniques. The GaN/AlN system appears to be a model system for a comparison between theory and experiments as interdiffusion between GaN and AlN is negligible. We verify that for the case of a three-dimensional system, such as a QD, the biaxial strain approximation is not valid. Furthermore, we demonstrate that the presence of QDs induces a modulation in the strain state of the AIN barriers which is the driving force for the vertical alignment of the GaN QDs in the AlN matrix. (C) 2005 American Institute of Physics.
\end{abstract}

[DOI: $10.1063 / 1.2123394]$

During the last decade, semiconductor quantum-dot (QD) heterostructures have been the subject of extensive research due to their unique electronic and optical properties. ${ }^{1}$ In particular, nitride QDs, have potential applications in optoelectronic devices, either in the visible or ultraviolet energy range. ${ }^{2}$ Moreover, with their piezoelectric characteristics, nitride QDs have particular behavior. The piezoelectric polarization, which has a magnitude comparable to the spontaneous polarization, induces a significant redshift of the luminescence lines of GaN/AlN QD heterostructures. Therefore, the knowledge of the strain distribution in QDs is essential to understand the emission wavelength and to expect to tune this wavelength to the desired application.

Despite the large number of reports on the growth and/or optical/structural characterization of wurtzite GaN/AIN QD, only a few theoretical studies address the strain state of these nanostructures. ${ }^{3,4}$ From the experimental viewpoint, Raman spectroscopy measurements ${ }^{5}$ provide information on the average strain of the GaN and AlN in the structures, indicating that the QDs are almost completely strained by the matrix, and the AlN is under a slight tensile strain. Anomalous grazing-incident $\mathrm{x}$-ray diffraction experiments resolve two strain states in the AlN matrix ${ }^{6}$ but the results were not compared to detailed elastic calculations.

In this letter, we investigate the two-dimensional strain distribution in vertically correlated GaN QD structures embedded in an AlN matrix by combining experimental data from high-resolution transmission electron microscopy (HRTEM) with elastic theoretical calculations.

Wurtzite GaN/AIN QD superlattices (SLs) with metal polarity, were grown on a $6 \mathrm{H}-\mathrm{SiC}$ substrate by plasmaassisted molecular beam epitaxy in a MECA2000 chamber equipped with standard effusion cells for $\mathrm{Al}$ and $\mathrm{Ga}$. Active nitrogen was produced in a radio-frequency plasma cell. Prior to the growth of the SL structure, a $0.5 \mu \mathrm{m}$ thick AlN buffer layer was deposited. The SL consists of 80 periods of

\footnotetext{
${ }^{\text {a) }}$ Author to whom correspondence should be addressed; permanent address: Laboratoire de Spectrométrie Physique, Université Joseph FourierGrenoble I, 140 Avenue de la physique, BP 87, 38402 Saint Martin d'Hères, France; electronic mail: eirini.sarigiannidou@tiscali.fr
}

GaN QD layers with $10 \mathrm{~nm}$ thick AlN barriers. The growth of GaN on AlN was made under N-rich conditions, by depositing an amount of about 6 monolayers (ML) of GaN. Under these conditions, the growth starts layer by layer, leading to the deposition of a $2 \mathrm{ML} \mathrm{GaN}$ wetting layer (WL), rapidly followed by the formation of three-dimensional islands (Stranski-Krastanov growth mode). ${ }^{7}$ The dots present truncated pyramidal shape with $\{10-13\}$ facets and an average height and base diameter of $4 \mathrm{~nm}$ and $30 \mathrm{~nm}$, respectively, as measured by HRTEM and atomic force microscopy. The QDs are vertically aligned along the [0001] growth direction. Transmission electron microscopy (TEM) cross section samples were prepared using the standard techniques of mechanical polishing and $\mathrm{Ar}^{+}$-ion milling.

A JEOL $3010 \mathrm{LaB}_{6}$ microscope equipped with a Gatan imaging filter was used for the energy-filtered TEM (EFTEM) experiments. A Ga elemental map of a series of GaN QDs is shown in Fig. 1. It was obtained using the "three-window" technique near the Ga $L_{2,3}$ edge. The spatial drift between the recorded images has been corrected using a cross correlation procedure. Thickness effects were also corrected. Within the resolution of the technique, the gallium signal drops to zero inside the AlN matrix. Therefore, we can consider the AlN/GaN interfaces as chemically abrupt and neglect $\mathrm{Al}-\mathrm{Ga}$ intermixing.

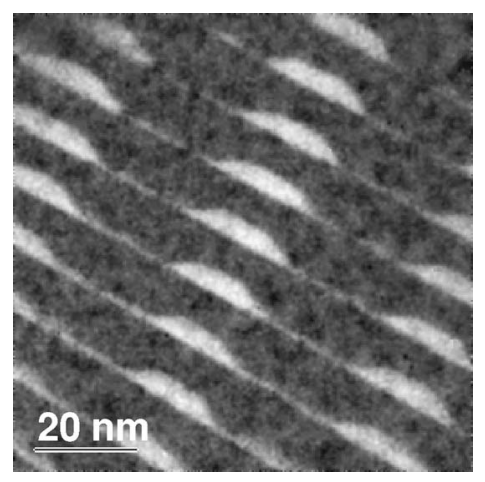

FIG. 1. Ga elemental map of a series of GaN QDs embedded inside an AIN matrix. Slit size $=15 \mathrm{eV}$, exposure time $=20 \mathrm{~s}$. The $\mathrm{Ga}$ signal drops to zero inside the AlN spacers. 

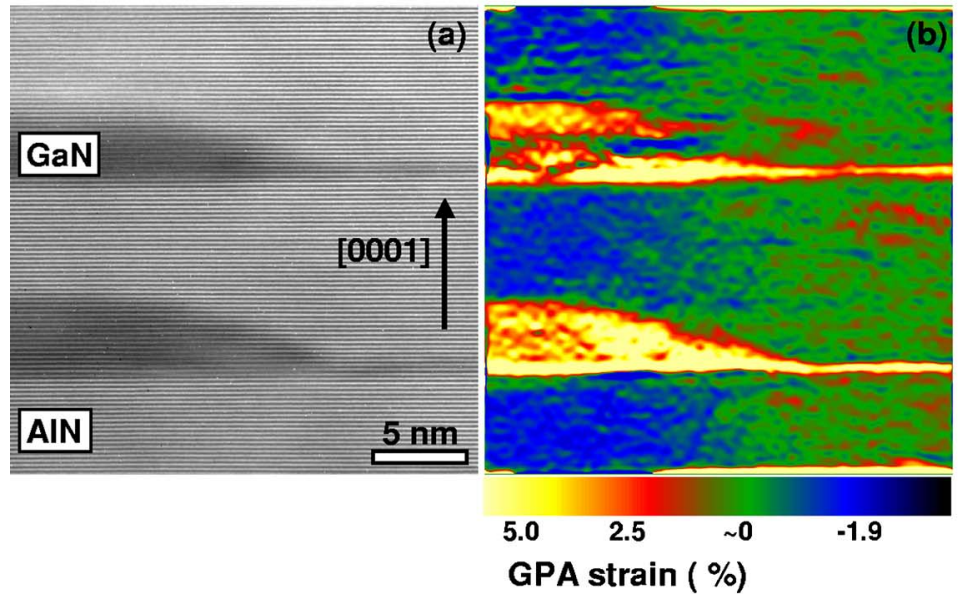

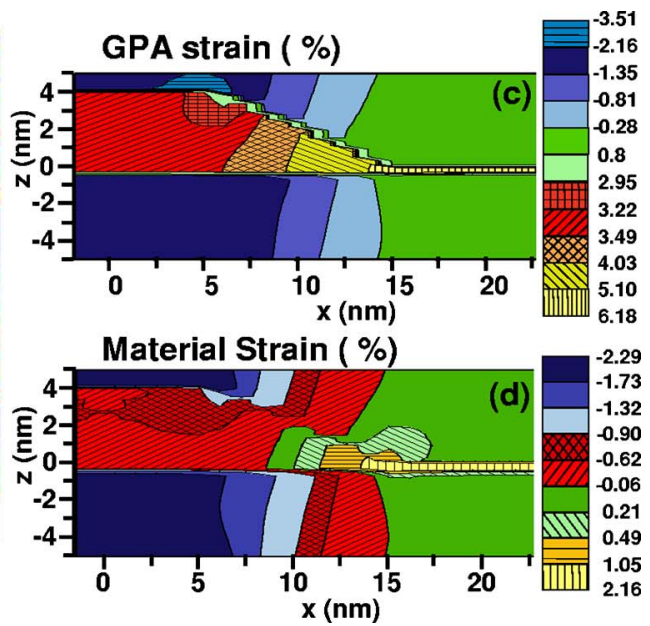

FIG. 2. (Color online) (a) Off-axis HRTEM image of two vertically aligned GaN QDs embedded inside an AlN matrix. Only the (0002) planes are visible. (b) Corresponding to (a) experimental $e_{z z}$ GPA strain map calculated using a Gaussian mask of size $\sim \mathrm{g} / 5$ around the 0002 reflection. (c) Calculated two-dimensional map of the GPA strain in the GaN/AIN QDs superlattice. (d) Calculated two-dimensional material strain map.

A JEOL4000EX electron microscope was used for HRTEM observations. For the measure of strain in the growth direction, HRTEM images were taken in a $\langle 5,-1,-4,0\rangle$ direction, as it has been shown that "off-axis" orientations improve the signal-to-noise ratio. ${ }^{8}$ For the measure of strain in a direction perpendicular to the growth direction, HRTEM images taken along a $\langle 2,-1,-1,0\rangle$ direction were used. Strain field maps were obtained by geometrical phase analysis (GPA) of HRTEM images. ${ }^{9,10}$ In our HRTEM measurements, we did not take into account the thin foil relaxation effect. ${ }^{11}$ We observe no significant gradients in the relative deformation as a function of the sample thickness in different examined images, far enough from both the sample surface and the buffer layer, which confirms that thin foil effects can be neglected in a first approximation.

The experimental data were compared to theoretical strain calculations that combine Fourier transform and Green's function techniques. ${ }^{3}$ In particular, the calculations presented here are based on solving the equations of the continuous linear elasticity theory for a periodic infinite SL of QDs presenting a truncated pyramidal shape with $\{10-13\}$ facets and a height and base diameter of $4 \mathrm{~nm}$ and $30 \mathrm{~nm}$, respectively. The hexagonal symmetry of the sample crystal structure is fully taken into account. Using the Green's func- tion technique, we find the Fourier transform of the strain tensor in the $k$ space. The spatial dependence of the strain tensor in real space is then found by summing the Fourier series. The elastic constant values for AlN and GaN were taken from the calculations by A. F. Wright. ${ }^{12}$ Different inplane distances between the GaN QDs, varying between 10 and $20 \mathrm{~nm}$, were considered, without significant effect on the obtained results.

Strain results were expressed using two different definitions of strain depending on the applied method. The GPA measures strain with respect to a reference area in the HRTEM image following the expressions:

$$
e_{z z}^{\mathrm{GPA}}(\mathbf{r})=\frac{c(\mathbf{r})-c^{\mathrm{ref}}}{c^{\mathrm{ref}}} ; \quad e_{x x}^{\mathrm{GPA}}(\mathbf{r})=\frac{a(\mathbf{r})-a^{\mathrm{ref}}}{a^{\mathrm{ref}}},
$$

where $c(\mathbf{r})$ and $a(\mathbf{r})$ are the lattice parameters at the pixel $\mathbf{r}$ of the HRTEM image, and $c^{\text {ref }}$ and $a^{\text {ref }}$ are the values averaged on the reference region of the image. This definition of strain, called here "GPA strain," is also known as GPA Lagrange strain. ${ }^{10}$ In this letter, we have chosen the AlN matrix situated between two WLs as the reference region.

In general, strain is measured with respect to the bulk lattice parameters of the material. Following this definition,
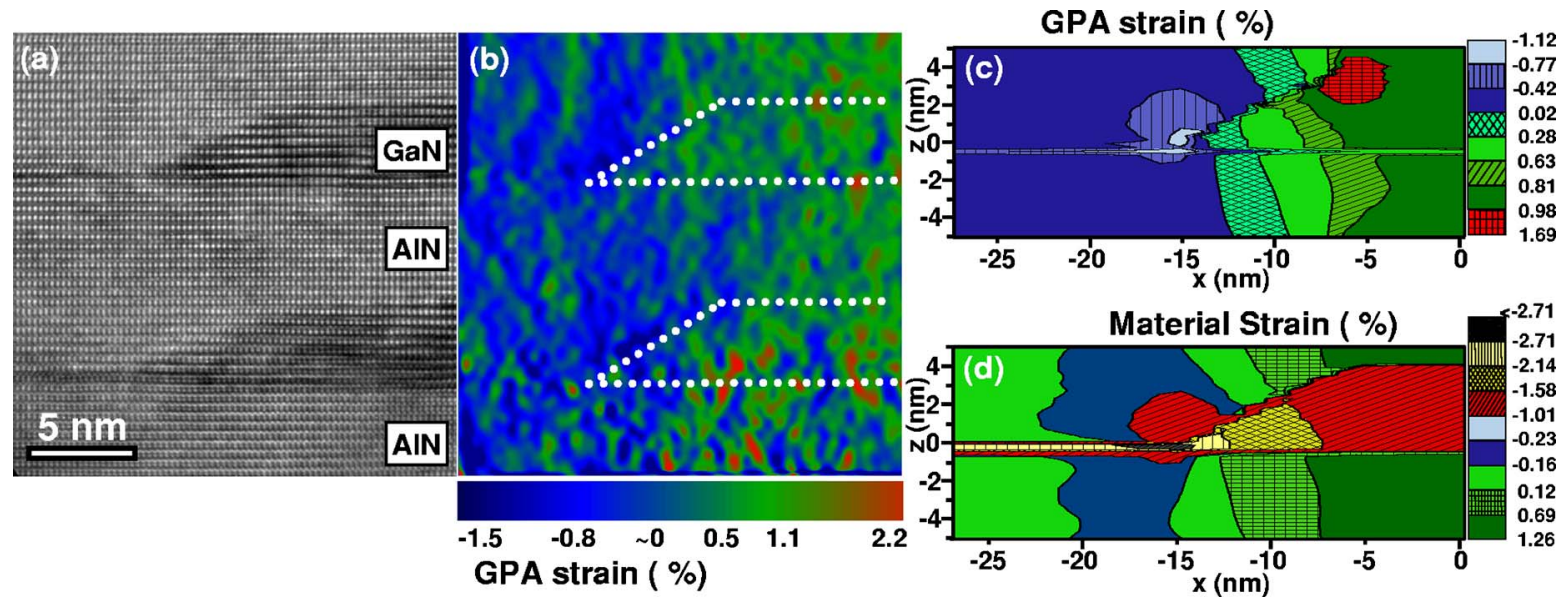

FIG. 3. (Color online) (a) High-resolution image of two GaN QDs of the superlattice taken along the [2,-1,-1,0] direction. (b) Corresponding GPA strain map calculated using the 01-10 reflection and by placing a Gaussian mask of size g/9 around it. (c) Calculated two-dimensional map of the GPA strain. (d) Calculated two-dimensional material strain map in the GaN/AIN QDs SL.

Downloaded 30 Mar 2009 to 131.227.178.132. Redistribution subject to AlP license or copyright; see http://apl.aip.org/apl/copyright.jsp 
TABLE I. Experimental and calculated results for the $e_{z z}$ and $e_{x x}$ strain components and $e_{z z} / e_{x x}$ ratio. The regions noted as AlN (WLs) and AlN (QDs) correspond to the AlN layer situated between two consecutive GaN WLs and two vertically aligned GaN QDs, respectively. GaN (WLs) is the wetting layer region. GaN (QDs) corresponds to the central region of the QD. The experimental values are averaged over the sample thickness.

\begin{tabular}{|c|c|c|c|c|c|c|c|}
\hline \multicolumn{4}{|c|}{$e_{z z}$} & \multicolumn{3}{|c|}{$e_{x x}$} & \multirow{3}{*}{$\begin{array}{c}e_{z z} / e_{x x} \\
\text { Material } \\
\text { strain } \\
\text { calcul. } \\
(\%)\end{array}$} \\
\hline & \multicolumn{2}{|c|}{ GPA strain } & \multirow{2}{*}{$\begin{array}{c}\text { Material } \\
\text { strain } \\
\text { calcul. } \\
(\%)\end{array}$} & \multicolumn{2}{|c|}{ GPA Strain } & \multirow{2}{*}{$\begin{array}{c}\text { Material } \\
\text { strain } \\
\text { calcul. } \\
(\%)\end{array}$} & \\
\hline & $\begin{array}{c}\text { Exp. } \\
( \pm 0.4 \%)\end{array}$ & $\begin{array}{c}\text { Calcul. } \\
(\%)\end{array}$ & & $\begin{array}{c}\text { Exp. } \\
( \pm 0.6 \%)\end{array}$ & $\begin{array}{c}\text { Calcul. } \\
(\%)\end{array}$ & & \\
\hline AlN (WLs) & $\approx 0$ & 0 & 0.14 & $\approx 0$ & 0 & -0.13 & -1.08 \\
\hline AlN (QDs) & -1.9 & -1.92 & -1.78 & 0.9 & 1.00 & 0.87 & -2.04 \\
\hline GaN (WLs) & 5.1 & 5.54 & 1.55 & $\approx 0$ & -0.03 & -2.57 & -0.6 \\
\hline GaN (QDs) & 2.7 & 3.31 & -0.60 & 0.9 & 1.18 & -1.39 & 0.4 \\
\hline
\end{tabular}

what we hereafter call "material strain" is equal to:

$$
e_{z z}^{\mathrm{mat}}(\mathbf{r})=\frac{c(\mathbf{r})-c_{\mathrm{Bulk}}}{c_{\mathrm{Bulk}}} ; \quad e_{x x}^{\mathrm{mat}}(\mathbf{r})=\frac{a(\mathbf{r})-a_{\mathrm{Bulk}}}{a_{\mathrm{Bulk}}},
$$

where $c_{\mathrm{Bulk}}$ and $a_{\mathrm{Bulk}}$ are the lattice parameters of the bulk material. GPA strain can be deduced from the material strain if the absolute value of $c^{\text {ref }}$ is known:

$$
\left[1+e_{z z}^{\mathrm{GPA}}(\mathbf{r})\right]=\left[1+e_{z z}^{\mathrm{mat}}(\mathbf{r})\right] \frac{c_{\mathrm{Bulk}}}{c_{\mathrm{ref}}} .
$$

Figures 2 and 3 respectively illustrate the analysis of the $e_{z z}$ and $e_{x x}$ strain components. Table I summarizes the average values of GPA and material strains in the main areas of the structures. In both directions, we notice the good agreement between the experimental and calculated GPA strain values. The strain extracted from HRTEM images are averaged over the sample thickness. Therefore, the presence of the matrix around the QD might result in an underestimation of the lattice parameters in the GaN QDs, particularly in the top and the facet areas. However, in the SL under study, the sample thickness is similar to the QD base diameter $(\sim 30 \mathrm{~nm})$. As a consequence, the influence of the matrix in our measurements should not be significant.

For the interpretation of the results, lets us recall that, in wurtzite layers under biaxial stress, the relation between the in-plane strain and the strain along the $c$ axis is given by the Poisson modulus: $e_{z z} / e_{x x}=-2 C_{13} / C_{33}(-0.58$ for AlN and -0.51 for $\mathrm{GaN})$. We notice that the GaN WL roughly follows a biaxial deformation, which is not the case for the $\mathrm{GaN}$ in the QDs due to the compressive hydrostatic stress imposed by the AlN matrix. Indeed, in our TEM image in Fig. 3(b), we observe that the GaN in the QDs is completely strained on the AlN barriers in the $a$ direction, while in Fig. 2(b) we observe a slight compression of the $c$ axis. Experimental values in GaN are slightly lower than calculated values. This is due to the faint relaxation of the stress at the free surface present in a TEM sample.

Regarding the AlN spacers, we observe two different strain states corresponding to the AlN situated between WLs (almost fully relaxed) and the AlN spacers between vertically correlated QDs (under tensile strain). An indication of the magnitude of the lateral stress induced by the QDs is the fact that the deformation of the AlN is not biaxial either between the QDs or between WLs. It is important to notice that the modulation in the AlN lattice parameters is not due to any $\mathrm{Ga}$ diffusion as proven by EFTEM experiments (Fig. 1).
All of these strain measurements demonstrate that the dominant mechanism responsible for the vertical correlation of GaN/AlN QDs is the inhomogeneous strain in the AlN matrix, and neither $\mathrm{Ga}-\mathrm{Al}$ interdiffusion nor the presence of threading dislocations. This vertical correlation mechanism has been described theoretically by Tersoff et al. ${ }^{13}$ It also explains the vertical alignment for the case of the Ge/Si QD system, whereas in the InAs/GaAs QD system the inhomogeneous strain in the GaAs matrix has sometimes been attributed to In diffusion. ${ }^{14}$ In our case, EFTEM experiments excluded such a possibility.

In conclusion, using HRTEM measurements in combination with theoretical calculations, the strain distribution in a GaN/AlN QD SL has been investigated. The GaN WL roughly follows a biaxial deformation, which is not the case for the GaN in the QDs due to the compressive hydrostatic stress imposed by the AlN matrix. We have experimentally identified the mechanism responsible for the vertical correlation of GaN/AIN QDs, which is the local strain induced in the AlN matrix by the presence of dots.

Thanks to Dr. P. Bayle-Guillemaud for her useful help during the EFTEM experiments.

${ }^{1}$ D. Bimberg, M. Grundmann, and N. N. Ledentsov, Quantum Dot Heterostructures (Wiley, Chichester, 1998).

${ }^{2}$ S. Nakamura, S. Pearton, and G. Fosol, The Blue Laser Diode: The Complete Story (Springer, Heidelberg, 2000).

${ }^{3}$ A. D. Andreev and E. P. O'Reilly, Phys. Rev. B 62, 15851 (2000).

${ }^{4}$ V. Ranjan, G. Allan, C. Priester, and C. Delerue, Phys. Rev. B 68, 115305 (2003).

${ }^{5}$ J. Gleize, F. Demangeot, J. Frandon, M. A. Renucci, M. Kuball, B. Damilano, N. Grandjean, and J. Massies, Appl. Phys. Lett. 79, 686 (2001).

${ }^{6}$ V. Chamard, T. Schülli, M. Sztucki, T. H. Metzger, E. Sarigiannidou, J. L. Rouvière, M. Tolan, C. Adelmann, and B. Daudin, Phys. Rev. B 69, 125327 (2004).

${ }^{7}$ B. Daudin, F. Widmann, G. Feuillet, Y. Samson, M. Arlery, and J. L. Rouvière, Phys. Rev. B 56, R7069 (1997).

${ }^{8}$ J. L. Rouvière, P. Bayle-Guillemaud, G. Radtke, S. Groh, and O. Briot, Inst. Phys. Conf. Ser. 169, 17 (2001).

${ }^{9}$ M. J. Hÿtch, E. Snoeck, and R. Kilaas, Ultramicroscopy 74, 131 (1998).

${ }^{10}$ J. L. Rouvière and E. Sarigiannidou, Ultramicroscopy (to be published).

${ }^{11}$ M. M. J. Treacy, J. M. Gibson, and A. Howie, Philos. Mag. A 51, 389 (1985).

${ }^{12}$ A. F. Wright, J. Appl. Phys. 82, 2833 (1997).

${ }^{13}$ J. Tersoff, C. Teichert, and M. G. Lagally, Phys. Rev. Lett. 76, 1675 (1996).

${ }^{14}$ Q. Xie, A. Madhukar, P. Chen, and N. P. Kobayashi, Phys. Rev. Lett. 75, 2542 (1995). 